\title{
Energetic Gamma-Ray Emission from Solar Flares
}

\author{
Ervin Kafexhiu ${ }^{1}\left(\mathbb{0}\right.$, Carlo Romoli ${ }^{1,2}$, Andrew M. Taylor $^{3}$, and Felix Aharonian ${ }^{1,2}$ \\ ${ }^{1}$ Max-Planck-Institut für Kernphysik, Saupfercheckweg 1, D-69117 Heidelberg, Germany; ervin.kafexhiu@mpi-hd.mpg.de \\ ${ }^{2}$ Dublin Institute for Advanced Studies, 31 Fitzwilliam Place, Dublin 2, Ireland \\ ${ }^{3}$ DESY, D-15738 Zeuthen, Germany \\ Received 2018 March 7; revised 2018 July 27; accepted 2018 August 1; published 2018 September 11
}

\begin{abstract}
Recent advances in the $\gamma$-ray observations of solar flares by the Fermi satellite demand revisions in the hadronic $\gamma$-ray flux computation below $1 \mathrm{GeV}$. In this work, we utilize recently updated pion production cross sections, along with an accurate description of low-energy nuclear interactions. Applying these new interaction descriptions to model the Fermi Large Area Telescope solar flare data, we infer primary particle spectral parameters. Application of this new cross section description leads to significantly different spectral parameters compared to those obtained previously. Furthermore, the inclusion of nuclei in these calculations leads to a primary spectrum that is generally harder than that required from proton-only considerations. Finally, the flare data at lower MeV energies, detected by the Fermi Gamma-ray Burst Monitor, are shown to provide additional low-energy spectral information.
\end{abstract}

Key words: gamma rays: general - Sun: flares - Sun: X-rays, gamma rays

\section{Introduction}

Solar flares are powerful outburst phenomena observed in the solar atmosphere, recorded to release an energy of up to $10^{33}$ erg in short time $\left(10^{2}-10^{3} \mathrm{~s}\right)$ intervals (see, e.g., Hudson \& Willson 1983; Kopp et al. 2005). Their energy source is believed to be the magnetic energy stored in the solar corona, released through magnetic reconnection. During these events, plasma heating up to $\mathrm{keV}$ temperatures and ion (electron) acceleration up to energies of tens of $\mathrm{GeV}$ (hundreds of $\mathrm{MeV}$ ) are observed. The largest solar flares are also associated with coronal mass ejections (CMEs); for a review, see, e.g., Aschwanden (2002), Benz (2008), and Fletcher et al. (2011).

A significant fraction of the energy released during these events has been thought to pass into nonthermal particles, with electrons dominating over protons at low $(<\mathrm{MeV})$ energies (Aschwanden et al. 2017). Despite their low-energy dominance, hard X-ray and $\gamma$-ray emission studies indicate that the spectra of electrons are soft above $\mathrm{MeV}$ energies (see, e.g., Lin et al. 1982; Lin 1985). Furthermore, the $\gamma$-ray spectra of the brightest flares above $100 \mathrm{MeV}$ would require an extremely hard electron power-law spectrum $\left(J_{e} \sim E_{e}^{-\alpha}\right.$ for $\alpha<2$; Ajello et al. 2014) in order to be explained by an electron emission scenario. It is therefore natural to assume that hadrons dominate significantly over electrons at high energies, such that the $\gamma$-ray emission above $100 \mathrm{MeV}$ is dominated by hadronic $\gamma$-ray production.

Solar flare $\gamma$-ray emission up to $100 \mathrm{MeV}$ was first detected from the GRS instrument on board the Solar Maximum Mission (Rieger et al. 1983). Following this, the Gamma-1 Telescope (Akimov et al. 1991) and EGRET on board the Compton Gamma-Ray Observatory (Kanbach et al. 1993) detected $\gamma$-rays emission above $100 \mathrm{MeV}$, reaching energies up to $2 \mathrm{GeV}$. The launch of the Fermi mission in 2008 started a new precision era in the study of high-energy $\gamma$-rays from the Sun.

The Fermi satellite has two detectors on board: the Fermi Gamma-ray Burst Monitor (Fermi-GBM) that is sensitive between $10 \mathrm{keV}$ and $30 \mathrm{MeV}$ (Meegan et al. 2009) and the Fermi Large Area Telescope (Fermi-LAT) that is sensitive between $20 \mathrm{MeV}$ and $300 \mathrm{GeV}$ (Atwood et al. 2009). The high statistics and energy resolution of the Fermi-LAT has allowed accurate determination of the $\gamma$-ray spectra from solar flares. Moreover, a recent release of the new PASS8 data has significantly increased the $\gamma$-ray sensitivity of the Fermi-LAT below $1 \mathrm{GeV}$, of particular importance for the study of ion distribution above $100 \mathrm{MeV} \mathrm{nuc}^{-1}$ in solar flare events.

Motivated by these recent improvements in solar flare observations, we implement here several improvements to the hadronic $\gamma$-ray production descriptions above $30 \mathrm{MeV}$. We first explore the application of new $p+p \rightarrow \pi$ cross sections, known to provide a particularly accurate description of the process for kinetic energies close to the threshold. We also implement and explore the additional consideration of subthreshold pion and $\gamma$-ray continuum production above $30 \mathrm{MeV}$, produced via nuclear interactions, both of which have previously been neglected.

The layout of this paper is as follows. In Section 2, we consider the Fermi-LAT data for four major solar flares and investigate the impact that the new PASS8 data have on two of these flares. In Section 3, we revise the $\gamma$-ray production cross sections and demonstrate explicitly the contributions of the subthreshold pions and the so-called hard photon channels, indicating the further impact that the consideration of different energetic particle abundances has on the final $\gamma$-ray spectrum. In Section 5, we discuss the primary spectra parameters and conclude with a summary of the main results.

\section{Solar Flare Data}

\subsection{Gamma-1 and Fermi-LAT Data}

We focus on six major solar flares. Five of them are detected by Fermi-LAT during solar cycle 24 between 2011 and 2014 . They are as follows: the 2011 March 7 and June 7 flares (Ackermann et al. 2014), the 2012 March 7 flare (Ajello et al. 2014), and the 2013 October 11 and the 2014 September 1 flares (Pesce-Rollins et al. 2015; Ackermann et al. 2017), analyzed using PASS7 and PASS8 instrument response functions (IRFs). In addition, we include the solar flare on 1991 June 15 (Leikov et al. 1993) detected by the Gamma-1 satellite. All of these flares are observed to emit $\gamma$-rays above $100 \mathrm{MeV}$, and they cover a wide energy interval from about 
$50 \mathrm{MeV}$ to several $\mathrm{GeV}$. Their spectral data carry similar features with a spectral energy distribution that peaks around $E_{\gamma} \approx 200 \mathrm{MeV}$. The 2012 March 7 flare, 2013 October 11 flare, and 1991 June 15 flare data are provided at different instances of their time evolution. These flares share a common feature, which is that their impulse phase is followed by a long and slowly varying $\gamma$-ray emission phase ${ }^{4}$ with $E_{\gamma}>100 \mathrm{MeV}$.

\subsection{Reanalysis of the Fermi-LAT Data Using the New PASS8 IRFs}

In $2015,^{5}$ the Fermi-LAT Collaboration released a new version of IRF called PASS8. In comparison with the previously released event reconstruction, there was a significant improvement in effective area, especially at energies below $1 \mathrm{GeV}^{6}$ that are particularly relevant for this study. For this reason, we assess the gain obtained with the reanalysis of the data focusing on two flares, namely, the 2011 June 7 and the 2013 October 11 flares. $^{7}$ These two flares were chosen because they were short and easy to analyze, given the fact that it was not needed to properly track the movement of the Sun in the field of view (Ackermann et al. 2014) or to use dedicated analysis due to the saturation of the anti-coincidence detector (Ajello et al. 2014).

The Fermi-LAT data were analyzed with the standard binned likelihood method in an energy range from $60 \mathrm{MeV}$ to $50 \mathrm{GeV}$ on photons of SOURCE class (evclass $=128$, evtype $=3$ ) in the same time intervals defined in Ackermann et al. (2014) and Pesce-Rollins et al. (2015). ${ }^{8}$ The region of interest (RoI) analyzed was a square region of $24^{\circ}$ size centered on the position of the Sun during the time of the flare. The analysis of a large portion of the sky is required due to the point-spread function (PSF) of the Fermi-LAT: while at $1 \mathrm{GeV}$ the $68 \%$ containment radius is roughly $1^{\circ}$, it increases toward lower energies with a value of almost $10^{\circ}$ for $60 \mathrm{MeV}$ photons. The localization of the centroid of the emission made use of the data above $100 \mathrm{MeV}$ to ensure a better PSF and was obtained using the standard tool gtfindsrc. For the 2011 June 7 flare, the Sun was close to the projected position of the Crab pulsar, so the centroid was extracted from a circle with a radius of $5^{\circ}$ to avoid contamination.

The emission of the Sun was modeled as a pointlike source centered in the centroid found previously, and the model file used in the gtlike routine included the diffuse model for the galactic and isotropic background as well. In the case of the 2011 flare, we added also the Crab pulsar. Because of this flare being in the vicinity of the galactic plane, the normalizations of these extra sources were left free. For the 2013 flare, the background models were instead fixed to the 3FGL catalog (Acero et al. 2015).

The new PASS8 reconstruction also allows for an extra feature to reduce the amount of systematic uncertainties by taking into account the energy dispersion matrix. This step is

\footnotetext{
4 The solar flare SF20131011 was a "behind the limb" flare, and its impulsive phase was not observed in $\gamma$-rays.

5 https://fermi.gsfc.nasa.gov/ssc/data/access/

6 http://www.slac.stanford.edu/exp/glast/groups/canda/lat_ Performance.htm

7 The flare SF20131011 was reanalyzed using the PASS8 reconstruction by Ackermann et al. (2017), but the results are not directly comparable because in the new analysis a different time interval was chosen.

8 The analysis followed the indications given by the Fermi-LAT collaboration in https://fermi.gsfc.nasa.gov/ssc/data/analysis/scitools/binned_likelihood_ tutorial.html.
}

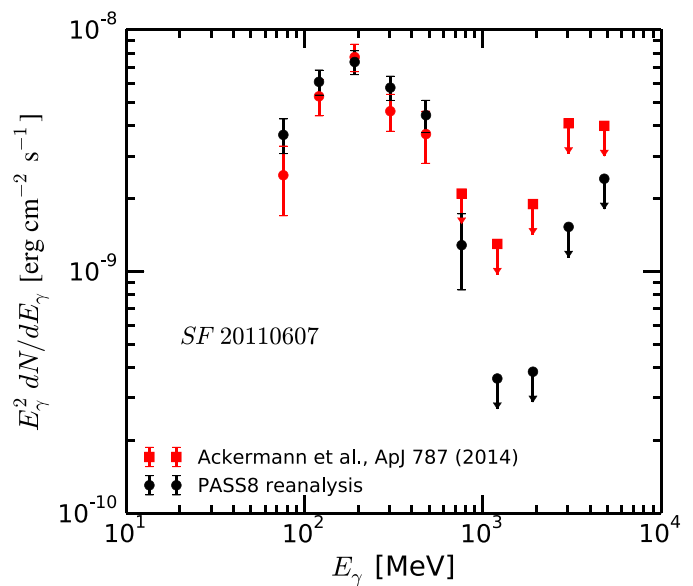

Figure 1. Comparison between the Fermi-LAT data of the 2011 June 7 solar flare reported in Ackermann et al. (2014; red squares) and the reanalysis made using the PASS8 IRFs (black circles). Besides the reduction of the size of the error bars, the upper limits above $10 \mathrm{GeV}$ are more constraining.

particularly important when analyzing, as in this case, energies below $100 \mathrm{MeV}{ }^{9}$

The SED points were computed following the procedure illustrated in Ackermann et al. (2014), fixing the power-law index at 2 and leaving free the normalization in each energy bin. The SEDs can be seen in Figure 1 for the 2011 flares, showing the improvement in the determination of the spectrum using the new software.

In Table 1, the results of the likelihood fit on the reanalyzed data are shown using a power law and a power law with exponential cutoff functions. The latter phenomenological function is statistically favored over a simple power law. The comparison with the already published data shows significant differences only for the power law with an exponential cutoff fit having the cutoff energy reconstructed at slightly lower energies.

\section{Gamma-Ray Production}

\subsection{Interaction Model}

We first consider a region in the solar atmosphere where accelerated primary particles interact with the ambient medium, producing secondary particles. Let $q_{s}\left(E_{s}, t\right)=d \dot{N}_{s} / d E_{s}$ be the secondary particle production rate per unit energy interval centered at energy $E_{s}$ at time $t$. The value of $q_{s}$ is computed as follows:

$$
q_{s}\left(E_{s}, t\right)=n_{t} \int_{E^{\text {th }}}^{\infty} d E f(E, t) v \frac{d \sigma}{d E_{s}}\left(E_{i}, E_{s}\right)
$$

where $n_{t}$ is the target medium number density, $E$ is the projectile energy, $E$ th is the threshold energy for the given reaction, $f(E, t)$ is the instantaneous energy distribution of the projectile particles in the interaction region, $v$ is the projectile speed, and $d \sigma / d E_{s}$ is the secondary particle production differential cross section for the specific process. It is clear from Equation (1) that the computation of $q_{s}$ in a given target medium density $n_{t}$, requires the primary particle energy

\footnotetext{
9 According to the official Fermi-LAT guidelines reported in https://fermi. gsfc.nasa.gov/ssc/data/analysis/documentation/Pass8_edisp_usage.html.
} 

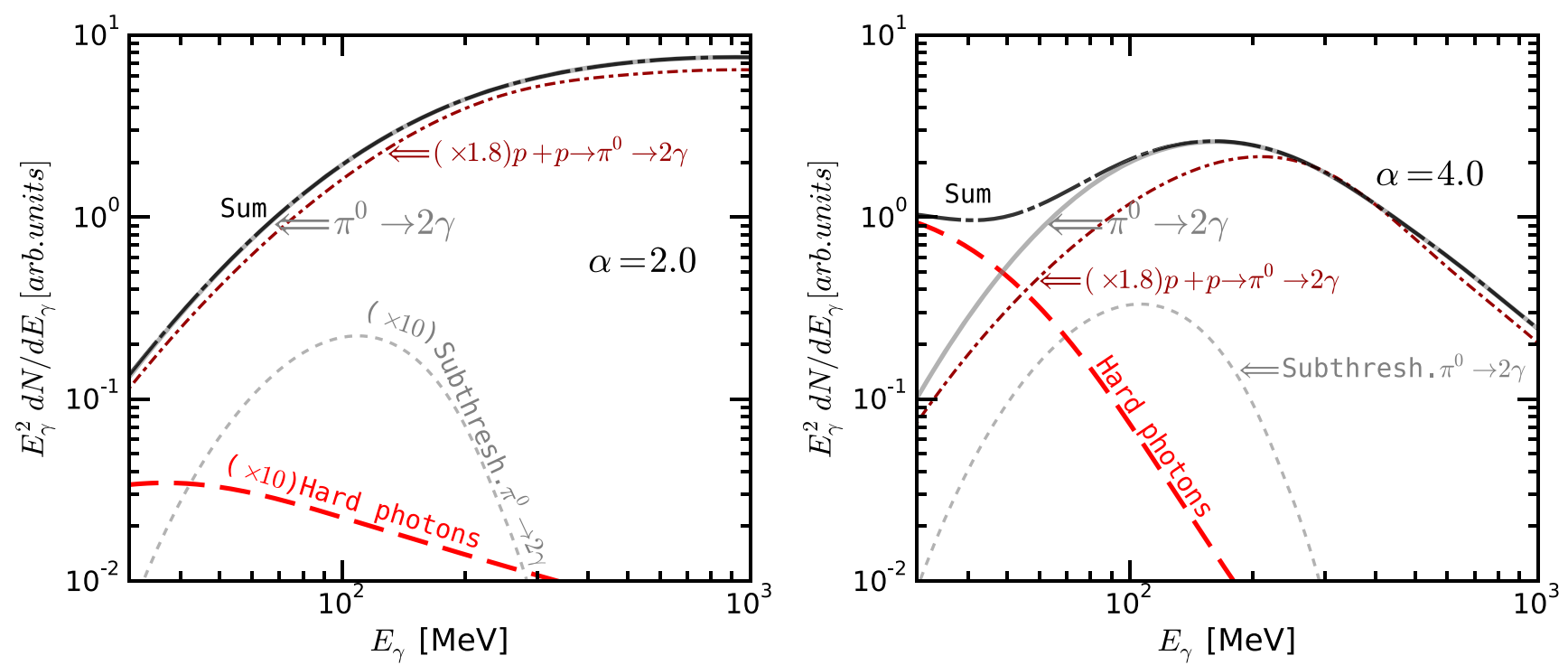

Figure 2. Nuclear $\gamma$-ray spectrum for $E_{\gamma}>30 \mathrm{MeV}$. The primary ion flux is a power-law function of kinetic energy per nucleon with $J_{i}=\left(f_{i} \times v\right) \sim T_{i}^{-\alpha}$ for $\alpha=2$ (left panel) and $\alpha=4$ (right panel); see Equation (1). The mass composition of the projectiles and target material are set to SEP (Reames 2014) and a solar composition (Lodders et al. 2009), respectively. The solid gray line shows the $\pi^{0} \rightarrow 2 \gamma$ contribution; the gray dashed line is only the contribution from the subthreshold pions. The red long-dashed line shows the contribution from hard photons. The black long-dashed-dotted line is the sum of the hard photon and $\pi^{0} \rightarrow 2 \gamma$ channels. For comparison, the $p+p \rightarrow \pi^{0} \rightarrow 2 \gamma$ is also shown (brown short-dashed-dotted line) and is multiplied by 1.8 (the nuclear enhancement factor) to compare with the nuclear spectrum. In the left panel, the contribution from the subthreshold pions and hard photons is small and is multiplied by a factor of 10 in the figure.

Table 1

Final Values of the Fit of the Two Solar Flares that Have Been Reanalyzed with PASS8 Data

\begin{tabular}{|c|c|c|c|c|c|}
\hline \multirow{2}{*}{ Data Set } & \multicolumn{2}{|c|}{ Power Law } & \multicolumn{3}{|c|}{ Power Law+Cutoff } \\
\hline & $\overline{\Phi_{100}\left(10^{-5} \mathrm{ph} \mathrm{cm}^{-2} \mathrm{~s}^{-1}\right)}$ & $\Gamma$ & $\overline{\Phi_{100}\left(10^{-5} \mathrm{ph} \mathrm{cm}^{-2} \mathrm{~s}^{-1}\right)}$ & $\Gamma$ & $E_{c}(\mathrm{MeV})$ \\
\hline 2011 Jun 7 & $2.62 \pm 0.17$ & $2.45 \pm 0.07$ & $3.22 \pm 0.21$ & $0.00 \pm 0.04$ & $103.6 \pm 6.6$ \\
\hline 2013 Oct 11 (a) & $14.9 \pm 0.4$ & $2.35 \pm 0.03$ & $18.4 \pm 0.5$ & $0.13 \pm 0.17$ & $125 \pm 11$ \\
\hline 2013 Oct $11(b)$ & $22.7 \pm 0.7$ & $2.37 \pm 0.03$ & $27.8 \pm 0.8$ & $0.22 \pm 0.17$ & $129 \pm 12$ \\
\hline
\end{tabular}

Note. The time range for the analysis of the 2011 June 7 solar flare is 07:47:40UT-08:23:40UT, whereas for the 2013 October 11 solar flare the times ranges are (a) 07:08:00UT-07:35:00UT and (b) 07:16:40UT-07:35:00UT. The parameter $\Phi_{100}$ indicates the flux above $100 \mathrm{MeV}$.

distribution $f$ and the specific process differential cross section and threshold energy.

Let us suppose that the energetic primary particles are injected in the interaction region with a rate per unit energy $Q$. Assuming that after being injected these particles can only lose energy or escape from the interaction region, their $f$ evolves with time (see, e.g., Ginzburg \& Syrovatskii 1964):

$$
\frac{\partial f}{\partial t}+\frac{\partial}{\partial E}\left(\frac{E f}{\tau_{\text {Eloss }}}\right)+\frac{f}{\tau_{\text {esc }}}=Q .
$$

Here, $\tau_{\text {Eloss }}$ is the energy-loss time, and $\tau_{\text {esc }}$ is the particle residence time in the interaction region.

Note that the solution of Equation (2) can be simplified for the two extreme limiting cases. In the first case, the escape of particles from the region dominates over energy losses (i.e., $\tau_{\text {esc }}<\tau_{\text {Eloss }}$ ), with the solution of Equation (2) being $f=Q \times \tau_{\text {esc }}$. In this case, the system is said to be in the thin target regime. In the second case, if one can neglect particle escape (i.e., $\tau_{\text {Eloss }}<\tau_{\text {esc }}$ ), the system is said to be in the thick target regime. In this regime, $f$ evolves with time until the rate of injected particles in the region balances the rate of particles removed from it via energy losses. At this equilibrium point, the evolution of $f$ reaches saturation.

\subsection{Gamma-Ray Production Cross Sections}

Solar flares can accelerate ions up to mildly relativistic energies. An accurate description of the $\gamma$-rays they produce thus requires accurate low-energy $\gamma$-ray production cross sections. Hydrogen is the most abundant element in the solar atmosphere. Energetic protons with energies above the threshold energy of $0.28 \mathrm{GeV}$, colliding with the ambient hydrogen, can produce $\gamma$-rays through pion production interactions. Nuclei on the other hand, although less abundant than hydrogen, can also significantly contribute to the final $\gamma$-ray spectrum. Unlike proton interactions, nuclei have the advantage of being able to produce pions via the so-called subthreshold pion production. Furthermore, they can also produce (below $0.28 \mathrm{GeV}$ nuc $^{-1}$ ) direct continuum emission at energies $E_{\gamma}>30 \mathrm{MeV}$ via the so-called hard photon production. These low-energy $\gamma$-ray production channels can be especially important for solar flares, as these produce steep primary particle spectra with low-energy cutoffs. These processes can significantly change the nuclear $\gamma$-ray spectral shape, compared to that produced for the simple proton-only case, and therefore should not be ignored.

The production cross section for $p+p \rightarrow \pi^{0} \rightarrow 2 \gamma$ has been recently parameterized in Kafexhiu et al. (2014). This new 

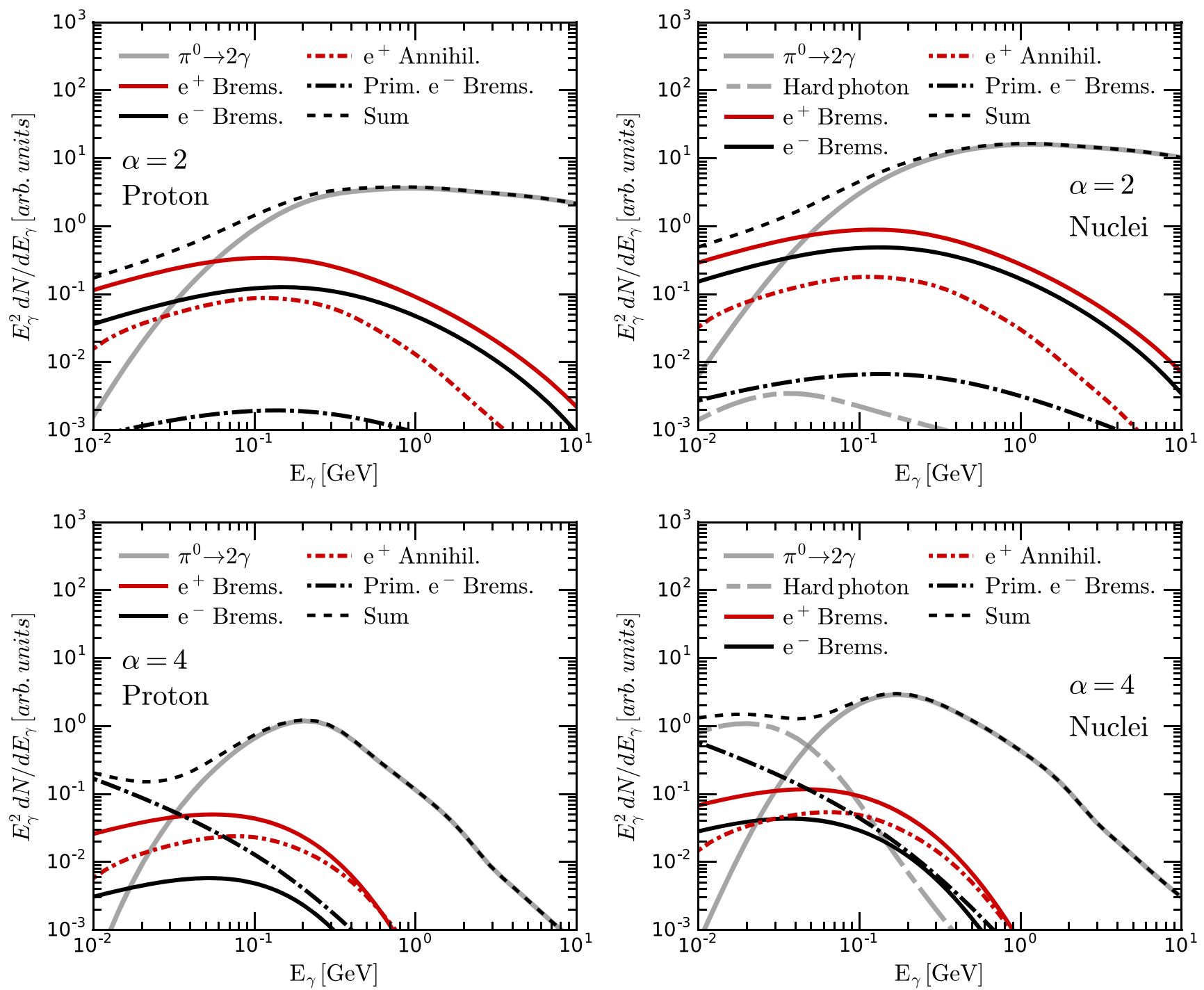

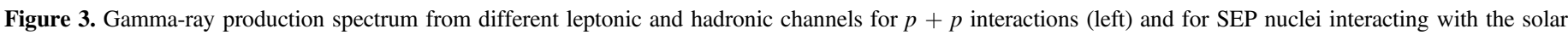

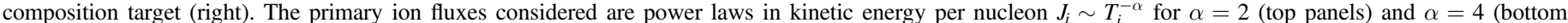

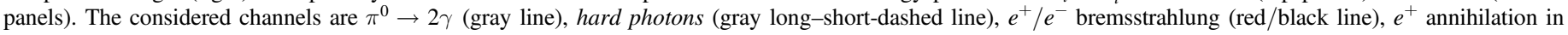

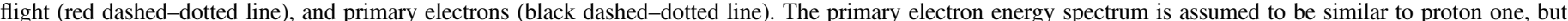
with $1 \%$ of their flux. The black dashed line is the sum of all channels.

parameterization is particularly useful for solar flare modeling, achieving high accuracies down to the kinematic threshold. It uses recent pion production experimental data for kinetic energies $T_{p}<2 \mathrm{GeV}$, and at higher collision energies utilizes a Monte Carlo description. We adopt here this parameterization to compute the $\gamma$ spectra from $p+p$ collisions.

The production cross sections for $p+p \rightarrow \pi^{ \pm}$at low collision energies near the kinematic threshold have also been recently parameterized in Yang et al. (2018). In this work, the charged pion energy distribution in the laboratory frame has been parameterized as a function of proton collision energy using the Geant4 toolkit (Agostinelli et al. 2003; Allison et al. 2006). The total cross sections of the charged pion production, on the other hand, are parameterized using publicly available experimental data; see Yang et al. (2018). We adopt this parameterization to compute the electron/positron spectra from $p+p$ collisions.

The $\gamma$-ray production cross sections for low-energy nuclei interactions, including the production of subthreshold pions and hard photons, have recently been parameterized in
Kafexhiu (2016). These parameterizations are based on publicly available experimental data and give simple and accurate analytical formulae that are valid for ion kinetic energies $T_{i} \leqslant 100 \mathrm{GeV} \mathrm{nuc}^{-1}$. We adopt here these formulae to compute the $\gamma$-ray and the $e^{ \pm}$spectra from all possible nuclear interactions.

To compute the electron and positron spectra from the decay of charged pions, produced via $p+p$ and nuclear interactions, we convolve the $\pi^{ \pm}$spectra with the $e^{ \pm}$energy distribution function for the monoenergetic pions (see, e.g., Scanlon \& Milford 1965; Dermer 1986a). The electron and positron spectra are then used to compute the $\gamma$-ray spectrum from the bremsstrahlung (Blumenthal \& Gould 1970) and annihilation in flight (Aharonian \& Atoyan 1981, 2000). We note that in the case of nuclei, bremsstrahlung emission has a $Z^{2}$ dependence and the annihilation in flight has a $Z$ dependence on the nuclear charge number $Z$.

Although not the primary focus of this paper, nuclear interactions can also produce $\gamma$-ray emission below $30 \mathrm{MeV}$. The most prominent contributors of this emission are the 

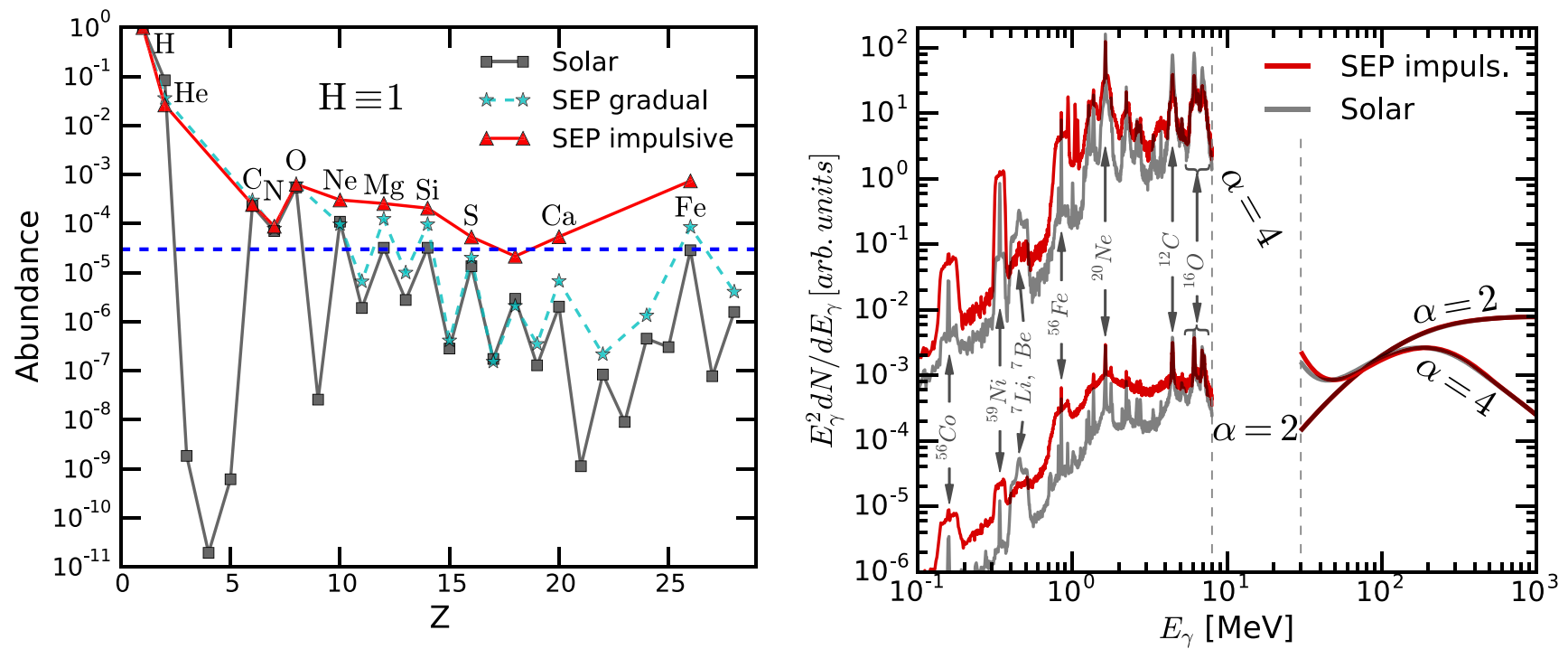

Figure 4. Left panel shows the elemental abundances for a solar composition (gray squares; Lodders et al. 2009) and solar energetic particles (SEPs) for gradual events (cyan stars) and impulsive events (red triangles; Reames 2014; Reames et al. 2014). The blue dashed line shows the threshold we use in our calculations to select the elements. Right panel shows the $\mathrm{MeV}$ and the $\mathrm{GeV} \gamma$-ray spectra for two different compositions of energetic particles: impulsive SEP (red line) and solar composition (gray line). The energetic particle fluxes are power laws in kinetic energy per nucleon with indices $\alpha=2$ and 4 . The final $\gamma$-ray spectra are normalized to have the same value at high energies. The elements that produce the strongest nuclear $\gamma$-ray lines are identified. The region between the vertical dashed gray lines is dominated by the compound and preequilibrium nuclear $\gamma$-ray continuum that has not been taken into account and that smoothly connects the nuclear lines with the higher-energy emission.
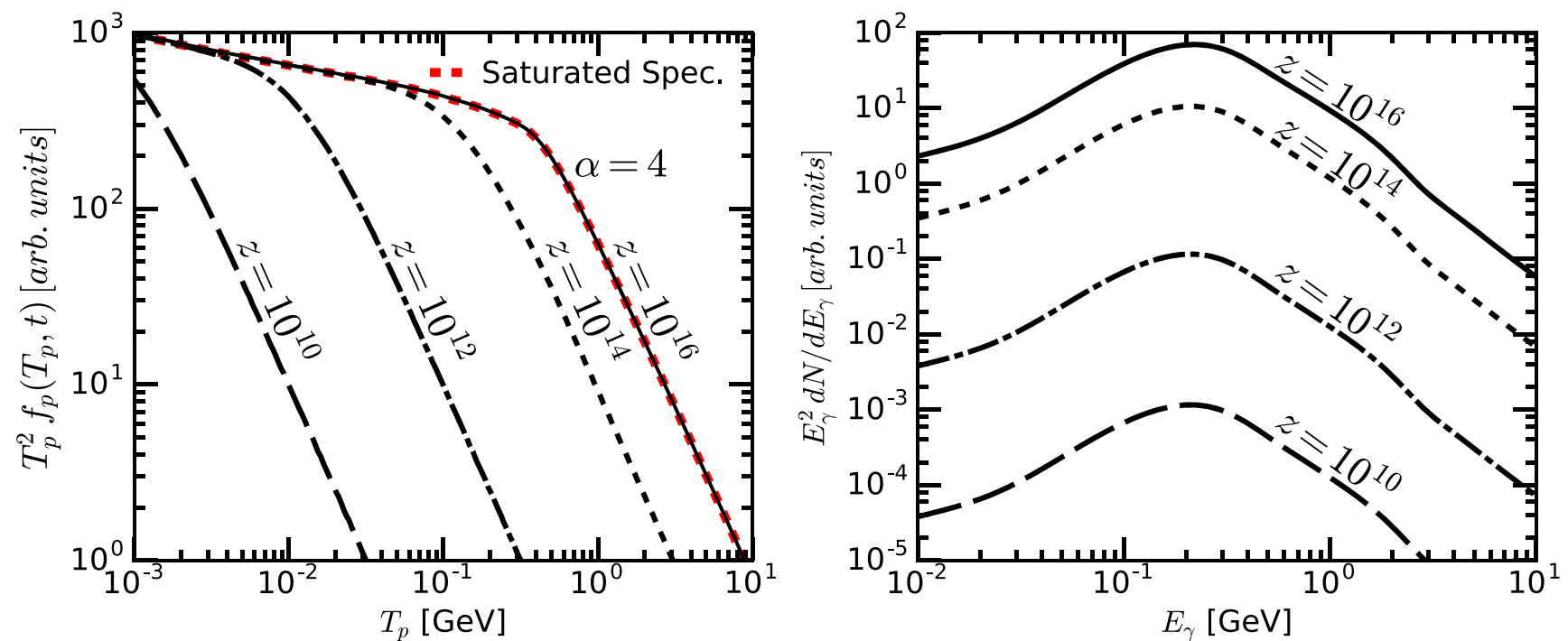

Figure 5. Evolution of the proton energy distribution $f_{p}$ and the resulting $\gamma$-ray spectra from $p+p$ interactions. The proton injection rate is considered as a power-law function of the form $Q_{p} \sim T_{p}^{-\alpha}$ with $\alpha=4$. The number density and the magnetic field strength are set to $n_{H}=10^{13} \mathrm{~cm}^{-3}$ and $B=100 \mathrm{G}$, respectively. The left panel shows the proton energy distribution evolution for different values of the parameter $z=n_{H} \times t$ that are set to $z=10^{10}$ (long-dashed line), $10^{12}$ (dashed-dotted line), $10^{14}$ (short-dashed line), and $10^{16} \mathrm{~cm}^{-3} \mathrm{~s}$ (solid line). For comparison, the saturated proton energy distribution is shown by a red dashed line, which is reached for $z \gtrsim 5 \times 10^{15} \mathrm{~cm}^{-3} \mathrm{~s}\left(t \gtrsim 5 \times 10^{2} \mathrm{~s}\right)$. Their corresponding $\gamma$-ray spectra are shown in the right panel.

nuclear $\gamma$-ray lines produced within the $0.1-10 \mathrm{MeV}$ energy interval; see, e.g., Ramaty et al. (1979). Their spectra have a strong dependence on the chemical composition of the target and projectile nuclei and the shape of the projectile particle spectrum below several hundreds of $\mathrm{MeV}$ nuc ${ }^{-1}$. For the calculation of the nuclear $\gamma$-ray line spectra, we adopt the Monte Carlo method described in Ramaty et al. (1979) and Kozlovsky et al. (2002), describing the nuclear $\gamma$-ray lines below $8 \mathrm{MeV}$. Note that recent developments in both the experimental data and numerical descriptions have increased the accuracy of the nuclear $\gamma$-ray line spectra (see, e.g., Belhout et al. 2007; Murphy et al. 2009; Benhabiles-Mezhoud et al. 2011; Kiener et al. 2012).

\section{Gamma-Ray Emission}

\subsection{Leptonic and Hadronic Channels}

We next apply the cross sections discussed above to the emission zone. As a first example we show the ensemble $\gamma$-ray emission from various channels following the interaction of energetic ions with the target gas. The flux of energetic ions is assumed to be a power-law function in kinetic energy per 


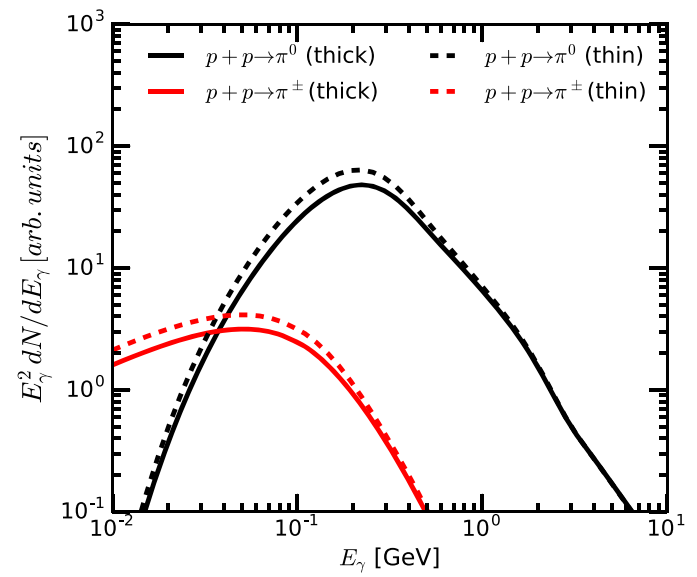

Figure 6. Gamma-ray spectrum from $p+p$ interactions for a thin target regime (dashed line) and a saturated spectrum for a thick target regime (solid line). The proton injection rate is assumed to be $Q_{p} \sim T_{p}^{-\alpha}$ for $\alpha=4$, and the number density and the magnetic field strength are set to $n_{H}=10^{13} \mathrm{~cm}^{-3}$ and $B=100 \mathrm{G}$. The black lines correspond to $\pi^{0} \rightarrow 2 \gamma$ decay, and the red lines correspond to electron and positron bremsstrahlung and annihilation in flight assuming saturated $e^{ \pm}$spectra.

nucleon, $J_{i}=f_{i} \times v \sim T_{i}^{-\alpha}$. Indexes of $\alpha=2$ and 4 are considered. The projectiles have a gradual solar energetic particle composition (gradual SEP; Reames 2014) and the target material has a solar composition (Lodders et al. 2009). We refer here to this abundance combination as "Nuclei." The resulting $\gamma$-ray spectra from both hadronic and leptonic emission are shown in Figures 2 and 3. In Figure 2, we explicitly show the $\gamma$-ray emission from neutral pion decay, including the subthreshold production and hard photon component of the hadronic emission. We note that the $\gamma$-ray spectrum for the proton-only interaction scenario is multiplied by 1.8 (the nuclear enhancement factor) to facilitate its comparison with the total nuclear spectrum.

Figure 3 includes the contribution from the leptonic $\gamma$-ray channels, such as $e^{ \pm}$bremsstrahlung, annihilation in flight, and primary electron bremsstrahlung. For this result, we consider the maximum possible contribution from electrons, obtained for the case of saturated (steady-state) $e^{ \pm}$spectra in the thick target regime. The $e^{ \pm}$injection rate is computed from the $\pi^{ \pm}$ decays, whereas, for the primary electrons, we assume that their injection rate is similar to that of protons, but normalized to only $1 \%$ of the proton flux.

It is clear from Figure 3 that the presence of nuclei can have significant effects on the total $\gamma$-ray spectrum below $E_{\gamma} \lesssim 200 \mathrm{MeV}$. These effects are larger for the soft energetic particle spectral case considered $(\alpha=4)$, for which proton-only interactions are unable to reproduce the $\gamma$-ray spectral shape. The additional inclusion of the leptonic channels further enhances such differences; see Figure 3. For the example case shown here, the total differences between the proton and nuclear spectral shape are less than $60 \%$ for $\alpha=2$ and a factor of 2 or more for the $\alpha=4$ case. It is therefore apparent that nuclear interactions produce notably different spectra compared to the proton-only case. Furthermore, the nuclear leptonic channels contribution is amplified by subthreshold $\pi^{ \pm}$production, an $e^{-} / e^{+}$ratio close to unity and the $Z^{2}$ dependence of the bremsstrahlung from the nucleus charge number $Z$. Note that unlike the low-energy proton-only interactions where the ratio $e^{-} / e^{+}$is close to zero, the low-energy nuclear interactions produce a comparable amount of $e^{ \pm}$due to isospin symmetry and having an equal number of protons and neutrons; see, e.g., Kafexhiu (2016).

\subsection{Chemical Composition of Energetic Particles}

We next explore the effect that different energetic particle chemical compositions have on the final $\gamma$-ray spectra. We adopt the same parameters as in the previous section, changing only the chemical composition of the energetic particles. Three of these compositions are considered: a solar composition (Solar), a gradual SEP, and an impulsive SEP composition (Reames 2014; Reames et al. 2014). These abundances are plotted in the left panel of Figure 4. As seen from the figure, the difference between a solar composition and a gradual SEP is not large. Therefore, when calculating the resulting $\gamma$-ray spectrum, we consider only energetic particles with a solar or impulsive SEP type composition. The $\gamma$-ray spectra for these cases are shown on the right panel of Figure 4.

In addition to the $\gamma$-ray continuum above $30 \mathrm{MeV}$, we have also computed the spectrum of nuclear $\gamma$-ray lines below $8 \mathrm{MeV}$. It is clear from Figure 4 that changes in the mass composition from solar to impulsive SEP do not notably effect the $\gamma$-ray spectrum above $30 \mathrm{MeV}$, the differences in the range 30-200 MeV being less than 40\%. However, the same changes in mass composition do have dramatic effects in the nuclear $\gamma$-ray line region. These differences originate from the fact that nuclei heavier than helium are more abundant for the impulsive SEP composition. Their excited emission subsequently suffers Doppler broadening effects. This results in the production of broad nuclear lines, which blend together to form a quasi-continuum for the SEP composition scenario.

\subsection{Proton Thick Target Emission}

Due to energy losses, the proton energy distribution evolves with time in the interaction region, until reaching saturation (steady state). Here, we compute this evolution and the resulting $\gamma$-ray spectra. We adopt the thick target regime for protons, with a power-law injection spectrum of the form $Q_{p}=\mathcal{N} \times T_{p}^{-\alpha}$ with $\alpha=4$ and $\mathcal{N}$, a normalization constant. The electrons and positrons produced via $\pi^{ \pm}$production are also injected into the interaction region and are also assumed to be in the thick target regime. Proton energy losses are dominated by ionization losses and inelastic collisions, whereas the energy losses for electrons are dominated by ionization, bremsstrahlung, and the synchrotron losses; see, e.g., Blumenthal \& Gould (1970) and Tanabashi et al. (2018). Since the proton energy losses are proportional to the target number density $n_{H}$, their energy distribution is better described by the quantity $z=n_{H} \times t$. Here, we assume that $n_{H}=10^{13} \mathrm{~cm}^{-3}$ and a magnetic field strength $B=100 \mathrm{G}$ as fiducial values for the solar atmosphere.

The left panel of Figure 5 shows the proton energy distribution evolution at four distinguishable epochs with $z=n_{H} \times t=10^{10}$, $10^{12}, 10^{14}$, and $10^{16} \mathrm{~cm}^{-3} \mathrm{~s}$, corresponding to evolution times of $t=10^{-3}, 10^{-1}, 10^{1}$, and $10^{3} \mathrm{~s}$, respectively. The proton energy distribution evolution can be understood in simple terms. When the evolution time is much smaller than the cooling timescale, the effect of losses is negligible. Therefore, the proton energy distribution has the same energy dependence as the injection rate $Q_{p}$, with the population of particles increasing linearly with time $f_{p} \sim Q_{p} t$; see, e.g., the $z=10^{10} \mathrm{~cm}^{-3}$ s curve. However, when 

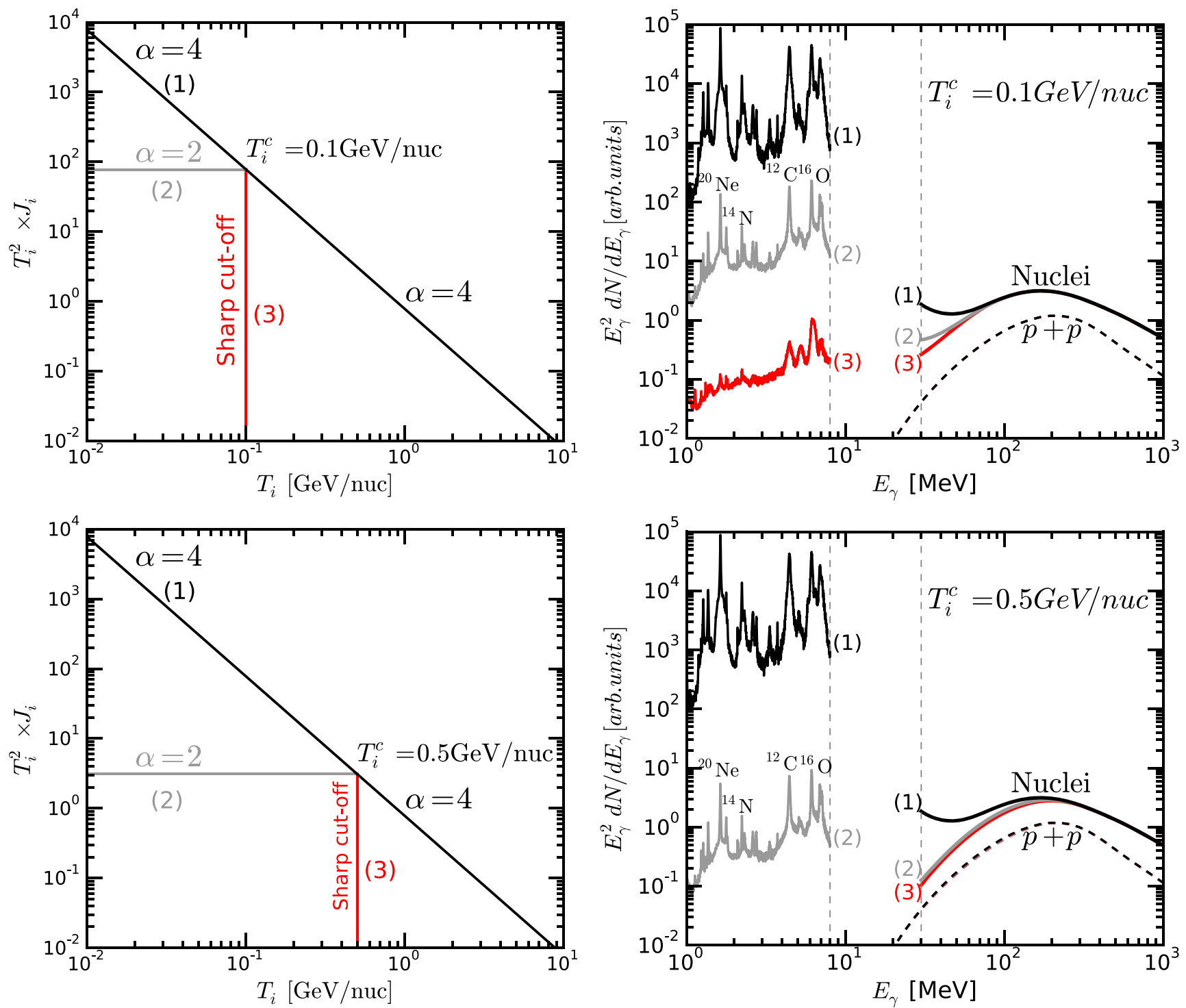

Figure 7. Energy distribution of ions (left panels) and their corresponding broadband $\gamma$-ray spectra (right panels). Left panels: energy distribution of ions with a power law on the projectile kinetic energy per nucleon $T_{i}$ with index $\alpha=4$ that has a break at lower energies at $T_{i}^{c}=0.1$ (top) and $0.5 \mathrm{GeV}$ nuc ${ }^{-1}$ (bottom). Curve (1) corresponds to the continuation of the power law with $\alpha=4$, curve (2) corresponds to $\alpha=2$ after the break, and curve (3) implies a sharp cutoff at the break energy. Right panels: corresponding $\gamma$-ray spectra due to $\pi^{0}$ production, hard photons, and nuclear $\gamma$-ray lines. The thin dashed lines show the $p+p$ contribution. The region between the vertical dashed gray lines is dominated by the compound and preequilibrium nuclear $\gamma$-ray continuum that has not been taken into account and that smoothly connects the nuclear lines with the higher-energy emission.

the evolution time $t$ becomes comparable with the cooling timescale, energy losses become important, shifting the highenergy population of particles toward lower energies. Consequently, $f_{p}$ starts to deviate from $Q_{p}$, with $f_{p}$ eventually reaching its saturation shape, after which its evolution ceases. For a steady injection rate, the proton energy distribution saturates for $z \gtrsim 5 \times 10^{15} \mathrm{~cm}^{-3} \mathrm{~s}$, corresponding for our example to $t \gtrsim$ $5 \times 10^{2} \mathrm{~s}$. Note that for an injection rate of the form $Q_{p} \sim T_{p}^{-\alpha}$ and energy losses of the form $\mathcal{P} \sim T_{p}^{\delta}$, the saturated energy distribution is also a power law $f_{p} \sim T_{p}^{-\beta}$ with $\beta=\alpha+\delta-1$. This explains the broken power-law shape of the proton energy distribution in the nonrelativistic region. The proton energy losses for $T_{p}>0.5 \mathrm{GeV}$ are dominated by inelastic collisions that have $\delta=1$; thus, $f_{p}$ has a similar energy dependence to $Q_{p}$ because $\beta=\alpha$. At lower energies, however, where ionization dominates energy losses, $\delta \approx-1$, the $f_{p}$ is a harder power law with $\beta \approx \alpha-2$. The subsequent maximum value of the energy break is reached for the saturate spectrum with $T_{p} \sim 0.4 \mathrm{GeV}$; see Figure 5.

The right panel of Figure 5 shows the resulting $\gamma$-ray spectra evolution produced for the setup described above, via protononly interactions. We note that similar computations for nuclei are not straightforward. Due to nuclear reactions, the nucleus number of a given species changes in the interaction region. The presence of nuclear spallation processes causes evolution of the nuclear abundances that must also be taken into account when calculating the nuclear $\gamma$-ray spectrum. Such considerations, however, are beyond the scope of this paper.

Figure 6 shows the contribution of the leptonic and hadronic channels to the final $\gamma$-ray spectra for extreme cases, namely, the thin target regime and the thick target regime, labeled "thick" and "thin" in the figure, respectively. Note that the 


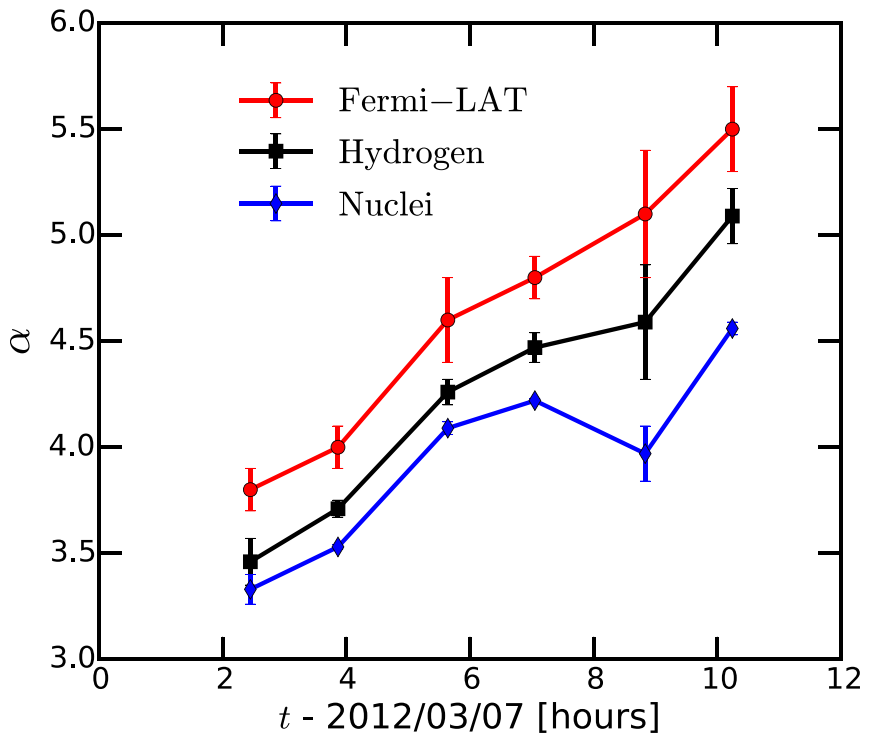

Figure 8. Time evolution of the power-law index $\alpha$ for the 2012 March 7 solar flare; see Table 2. The blue error bars show the results for nuclei (SEP interaction with solar composition target material), the black error bars show the results for pure hydrogen composition (using our updated $p+p$ cross sections; Kafexhiu et al. 2014; Yang et al. 2018), and the red error bars show the results from Ajello et al. (2014); see Table 2.

Table 2

The MCMC Results for the Primary Spectrum Power-law Index $\alpha$ for the Published Solar Flare Data that We Have Considered Here

\begin{tabular}{lccc}
\hline \hline Flare & Hydrogen & Nuclei & Fermi \\
\hline 2011 Mar 7 & $4.27_{-0.20}^{+0.22}$ & $3.80_{-0.09}^{+0.11}$ & $4.5_{-0.2}^{+0.2}$ \\
2011 Jun 7 & $4.12_{-0.43}^{+0.54}$ & $3.48_{-0.14}^{+0.19}$ & $4.3_{-0.3}^{+0.3}$ \\
2012 Mar 7 (a) & $3.46_{-0.11}^{+0.13}$ & $3.33_{-0.07}^{+0.09}$ & $3.8_{-0.1}^{+0.1}$ \\
2012 Mar 7 (b) & $3.71_{-0.04}^{+0.04}$ & $3.53_{-0.01}^{+0.02}$ & $4.0_{-0.1}^{+0.1}$ \\
2012 Mar 7 (c) & $4.26_{-0.06}^{+0.10}$ & $4.09_{-0.03}^{+0.03}$ & $4.6_{-0.2}^{+0.2}$ \\
2012 Mar 7 (d) & $4.47_{-0.07}^{+0.07}$ & $4.22_{-0.01}^{+0.01}$ & $4.8_{-0.1}^{+0.1}$ \\
2012 Mar 7 (e) & $4.59_{-0.27}^{+0.31}$ & $3.97_{-0.13}^{+0.14}$ & $5.1_{-0.3}^{+0.3}$ \\
2012 Mar 7 (f) & $5.09_{-0.13}^{+0.13}$ & $4.56_{-0.03}^{+0.03}$ & $5.5_{-0.2}^{+0.2}$ \\
2013 Oct 11 (a) & $3.98_{-0.24}^{+0.30}$ & $3.71_{-0.20}^{+0.21}$ & $3.8_{-0.2}^{+0.2}$ \\
2013 Oct 11 (b) & $3.88_{-0.22}^{+0.26}$ & $3.62_{-0.18}^{+0.19}$ & $3.7_{-0.2}^{+0.2}$ \\
2014 Sep 1 & $3.95_{-0.05}^{+0.06}$ & $3.71_{-0.03}^{+0.03}$ & $4.4_{-0.1}^{+0.1}$ \\
\hline 1991 Jun 15 (a) & $3.71_{-0.06}^{+0.07}$ & $3.51_{-0.04}^{+0.04}$ & $\cdots$ \\
1991 Jun 15 (b) & $3.81_{-0.10}^{+0.10}$ & $3.52_{-0.05}^{+0.05}$ & $\ldots$ \\
1991 Jun 15 (c) & $5.01_{-0.13}^{+0.14}$ & $3.93_{-0.03}^{+0.03}$ & $\ldots$ \\
\hline
\end{tabular}

Note. The "Hydrogen" are the results for pure hydrogen composition, whereas "Nuclei" are the results for the SEP interacting with a solar composition target material. The "Fermi" column quotes the index $\alpha$ values that are published in Fermi-LAT publications (Ackermann et al. 2014, 2017; Ajello et al. 2014; Pesce-Rollins et al. 2015). For the 2012 March 7 flare the time extended emission data are provided with starting times (a) 02:27:00UT, (b) 03:52:00UT, (c) 05:38:32UT, (d) 07:03:00UT, (e) 08:50:00UT, and (f) 10:14:32UT (Ajello et al. 2014). For the 2013 October 11 flare data are provided for the intervals (a) 07:16:40UT-07:35:00UT and (b) 07:08:00UT07:35:00UT (Pesce-Rollins et al. 2015). For the 1991 June 15 flare the time extended emission data are (a) 08:37:22-08:42:22, (b) 08:42:22-08:50:00, and (c) 08:50:00-09:00:14 (Leikov et al. 1993).

radiation from $e^{ \pm}$bremsstrahlung and annihilation in flight are calculated for their saturated spectral cases, corresponding to their maximal potential contribution. We recall that the

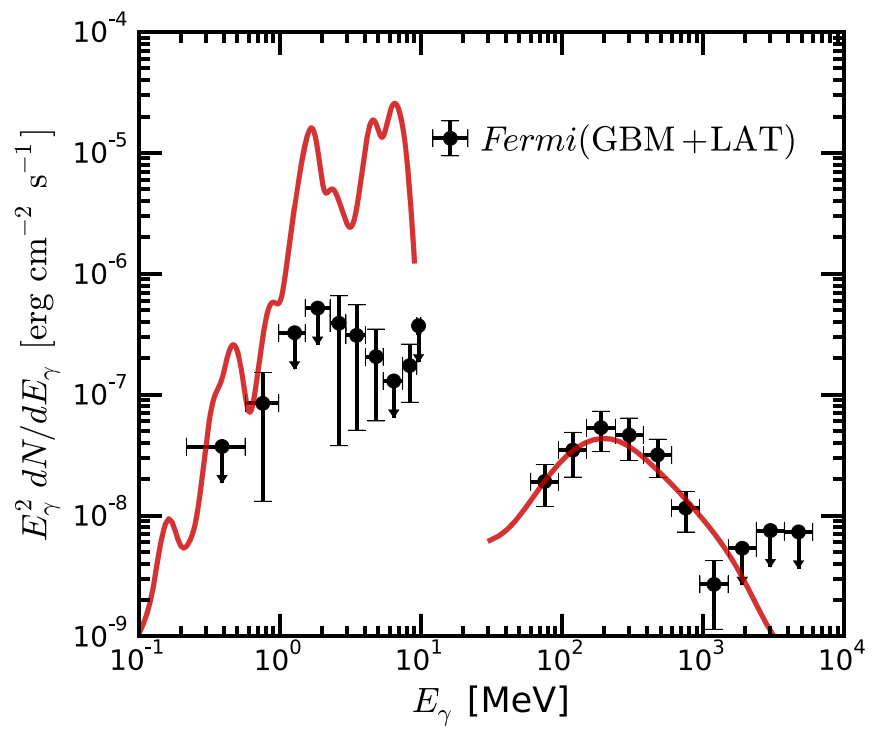

Figure 9. $\mathrm{MeV}$ and $\mathrm{GeV} \gamma$-ray spectra from the 2013 October 11 solar flare observed by the Fermi-GBM and the Fermi-LAT (Pesce-Rollins et al. 2015). The red line is the calculation using a power-law ion flux with index $\alpha=3.7$ derived by fitting the Fermi-LAT data. The $\gamma$-ray flux below $10 \mathrm{MeV}$ is calculated assuming a continuation of the power-law function toward lower energies. The flux below $10 \mathrm{MeV}$ is also smoothed to take into account the $10 \%$ energy resolution of the Fermi-GBM detector. The energetic ion abundances are set to SEP and for the target material to a solar composition.

bremsstrahlung and annihilation in flight for a thin target $e^{ \pm}$ regime can be negligible.

\subsection{Low-energy Spectra of Energetic Particles}

Here, we explore the effect that the low-energy spectral shape of the energetic particle spectra has on the $\gamma$-ray emission. We assume a thin target regime for simplicity, with the ion flux being described by a broken power law. We consider break energies of $T_{i}^{c}=100$ and $500 \mathrm{MeV} \mathrm{nuc}^{-1}$. The high-energy part of the power law has a fixed index of $\alpha=4$. The shape below the break energy is described by (1) a continuation of the $\alpha=4$ power law, (2) an $\alpha=2$ power law, and (3) a sharp low-energy cutoff.

The left panel of Figure 7 shows the energetic particle spectra, whereas the right panel shows the respective $\gamma$-ray spectra. We do not include here the $\gamma$-ray production from secondary $e^{ \pm}$channels. It is clear from the figure that the lowenergy primary spectral shape has a dramatic effect on the $\gamma$-ray spectrum below $200 \mathrm{MeV}$, especially in the energy region of the nuclear $\gamma$-ray lines where the emissivities can change by orders of magnitude. These effects will be magnified if the solar composition is replaced by a heavier one. Unlike the nuclear interactions, the resulting radiation spectrum from proton-only interactions remains practically unchanged.

\section{Results and Discussion}

In this section, we show the energetic particle spectral parameters obtained by fitting the solar flare data described in Section 2. For this analysis we assume a thin target regime for ions and a thick target regime for the secondary electrons (i.e., adopting their saturated spectra). We also consider two chemical compositions, namely, a pure proton (hydrogen) and an SEP composition (nuclei), interacting with solar abundance target material. We note that changing the chemical 
composition of energetic particles from gradual to impulsive SEP or to a solar composition has negligible effects in the energy range $E_{\gamma}>50 \mathrm{MeV}$ relevant for the Fermi-LAT and Gamma-1 solar flare data; see Figure 4. We consider a primary ion flux described by a power-law function of the form $J_{i}=N \times T_{i}^{-\alpha}$, where the normalization constant $N$ and the power-law index $\alpha$ are free parameters. For exploring this spectral parameter space, we adopt the Goodman and Weare's affine invariant Markov Chain Monte Carlo Ensemble sampler (MCMC) as is implemented in Foreman-Mackey et al. (2013) and adopt the revised $\gamma$-ray production cross sections described in Section 3. The results of the analysis are summarized in Table 2.

We next compare the results obtained here for the hydrogen case with the same ones quoted in the Fermi-LAT publications. As seen in Table 2, the index $\alpha$ obtained in this work has significant deviations from the values quoted in the literature. These changes can be predominantly explained by the differences in the $p+p$ cross sections adopted between our revised parameterizations and the ones used in the Fermi-LAT publications (Dermer 1986b; Murphy et al. 1987).

Further significant differences are also seen when nuclei are considered. The index $\alpha$ required to fit the $\gamma$-ray data is systematically smaller for nuclei than for the hydrogen case; see Table 2. Thus, for nuclei, the same $\gamma$-ray data require a harder primary spectrum than the corresponding proton-only values. These contrasts in the primary particle parameter space are a reflection of their different $\gamma$-ray spectral shape for $E_{\gamma}<200 \mathrm{MeV}$.

Observations of the 2012 March 7 and 2013 October 11 solar flares by Fermi-LAT and the 1991 June 15 flare by Gamma-1 have provided $\gamma$-ray data at different instances during the evolution of the flares. Specifically, the analysis of the 2012 March 7 flare data suggests that the power-law index $\alpha$ increases with time; see Figure 8 .

For the 2013 October 11 flare, the Fermi-GBM data below $10 \mathrm{MeV}$ are also provided (Pesce-Rollins et al. 2015). Figure 9 shows the subsequent best-fit $\gamma$-ray spectrum to the Fermi-LAT data, with a low-energy comparison to the Fermi-GBM data for the Nuclei composition case. We assume here that the same functional form of the primary spectra fit to the Fermi-LAT data continues down to the lower energies relevant for nuclear $\gamma$-ray line production. As we can see in Figure 9, the $\gamma$-ray flux predicted from the soft pure power-law primary flux fits well the high-energy data, but overpredicts the $\mathrm{MeV} \gamma$-ray flux. Note, however, that in the thick target regime, ionization losses will harden the nonrelativistic part of the ion spectrum. The $\mathrm{MeV} \gamma$-ray fluxes predicted here may therefore be reduced, e.g., see Figure 7. Furthermore, for the Nuclei composition case, with the energetic particles interacting in the thick target regime, the evolution of the nuclear states due to spallation will further complicate this picture. Interestingly, such an evolution may offer a future opportunity to probe the nuclear residence times using the nuclear $\gamma$-ray line information.

Finally, we recall that our reanalysis of the 2011 June 7 and the 2013 October 11 data using the new PASS8 data shows improvements on the quality of the data by reducing the error bars and by adding one more data point around $1 \mathrm{GeV}$; see Figure 1. Despite this, the final primary spectra parameters required to fit the $\gamma$-ray data do not show significant changes.

\section{Conclusions}

The high-quality $\gamma$-ray solar flare observations carried out by Fermi-LAT data demands accurate modeling of this $\gamma$-ray emission above $30 \mathrm{MeV}$. In this work, we have revised hadronic $\gamma$-ray emission calculations for both protons and nuclei, taking into account the secondary electrons produced. Utilizing our recent updates to the hadronic $\gamma$-ray production cross sections for both protons and nuclei, the importance of the description of pion production close to threshold, nuclear subthreshold pion production, and hard photon emission are highlighted. Neglecting these processes is found to be considerably detrimental in the recovery of the underlying projectile particle spectrum using the Fermi-LAT $\gamma$-ray data.

\section{ORCID iDs}

Ervin Kafexhiu (1D https://orcid.org/0000-0003-1174-4729

\section{References}

Acero, F., Ackermann, M., Ajello, M., et al. 2015, ApJS, 218, 23 Ackermann, M., Ajello, M., Albert, A., et al. 2014, ApJ, 787, 15 Ackermann, M., Allafort, A., Baldini, L., et al. 2017, ApJ, 835, 219

Agostinelli, S., Allison, J., Amako, K., et al. 2003, NIMPA, 506, 250

Aharonian, F. A., \& Atoyan, A. M. 1981, PhLB, 99, 301

Aharonian, F. A., \& Atoyan, A. M. 2000, A\&A, 362, 937

Ajello, M., Albert, A., Allafort, A., et al. 2014, ApJ, 789, 20

Akimov, V. V., Afanassyey, V. G., Belaousov, A. S., et al. 1991, Proc. ICRC (Dublin), 22, 73

Allison, J., Amako, K., Apostolakis, J., et al. 2006, ITNS, 53, 270

Aschwanden, M. J. 2002, SSRv, 101, 1

Aschwanden, M. J., Caspi, A., Cohen, C. M. S., et al. 2017, ApJ, 836, 17

Atwood, W. B., Abdo, A. A., Ackermann, M., et al. 2009, ApJ, 697, 1071

Belhout, A., Kiener, J., Coc, A., et al. 2007, PhRvC, 76, 034607

Benhabiles-Mezhoud, H., Kiener, J., Thibaud, J.-P., et al. 2011, PhRvC, 83, 024603

Benz, A. O. 2008, LRSP, 5, 1

Blumenthal, G. R., \& Gould, R. J. 1970, RvMP, 42, 237

Dermer, C. D. 1986a, ApJ, 307, 47

Dermer, C. D. 1986b, A\&A, 157, 223

Fletcher, L., Dennis, B. R., Hudson, H. S., et al. 2011, SSRv, 159, 19

Foreman-Mackey, D., Hogg, D. W., Lang, D., \& Goodman, J. 2013, PASP, 125,306

Ginzburg, V. L., \& Syrovatskii, S. I. 1964, The Origin of Cosmic Rays (Oxford: Pergamon)

Hudson, H. S., \& Willson, R. C. 1983, SoPh, 86, 123

Kafexhiu, E. 2016, PhRvC, 94, 064603

Kafexhiu, E., Aharonian, F., Taylor, A. M., \& Vila, G. S. 2014, PhRvD, 90, 123014

Kanbach, G., Bertsch, D. L., Fichtel, C. E., et al. 1993, A\&AS, 97, 349

Kiener, J., Tatischeff, V., Benhabiles-Mezhoud, H., de Séréville, N., \& Belhout, A. 2012, JPhCS, 366, 012026

Kopp, G., Lawrence, G., \& Rottman, G. 2005, SoPh, 230, 129

Kozlovsky, B., Murphy, R. J., \& Ramaty, R. 2002, ApJS, 141, 523

Leikov, N. G., Akimov, V. V., Volzhenskaia, V. A., et al. 1993, A\&AS, 97, 345

Lin, R. P. 1985, SoPh, 100, 537

Lin, R. P., Mewaldt, R. A., \& Van Hollebeke, M. A. I. 1982, ApJ, 253, 949

Lodders, K., Palme, H., \& Gail, H.-P. 2009, LanB, 4, 712

Meegan, C., Lichti, G., Bhat, P. N., et al. 2009, ApJ, 702, 791

Murphy, R. J., Dermer, C. D., \& Ramaty, R. 1987, ApJS, 63, 721

Murphy, R. J., Kozlovsky, B., Kiener, J., \& Share, G. H. 2009, ApJS, 183, 142

Pesce-Rollins, M., Omodei, N., Petrosian, V., et al. 2015, ApJL, 805, L15

Ramaty, R., Kozlovsky, B., \& Lingenfelter, R. E. 1979, ApJS, 40, 487

Reames, D. V. 2014, SoPh, 289, 977

Reames, D. V., Cliver, E. W., \& Kahler, S. W. 2014, SoPh, 289, 3817

Rieger, E., Reppin, C., Kanbach, G., et al. 1983, Proc. ICRC (Bangalore), 18,338

Scanlon, J. H., \& Milford, S. N. 1965, ApJ, 141, 718

Tanabashi, M., Hagiwara, K., Hikasa, K., et al. 2018, PhRvD, 98, 030001

Yang, R., Kafexhiu, E., \& Aharonian, F. 2018, A\&A, 615, A108 